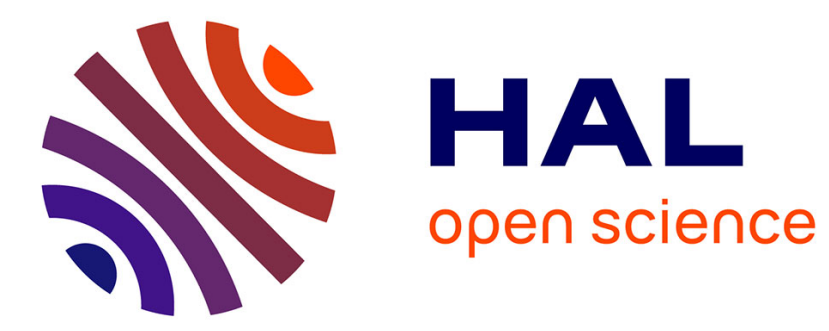

\title{
Light-front and conformal field theories in two dimensions
}

\author{
Pierre Grangé, Ernst Werner
}

\section{To cite this version:}

Pierre Grangé, Ernst Werner. Light-front and conformal field theories in two dimensions. Mod.Phys.Lett.A, 2018, 33 (22), pp.1850119. 10.1142/S0217732318501195 . hal-01861937

\section{HAL Id: hal-01861937 \\ https://hal.science/hal-01861937}

Submitted on 13 Sep 2018

HAL is a multi-disciplinary open access archive for the deposit and dissemination of scientific research documents, whether they are published or not. The documents may come from teaching and research institutions in France or abroad, or from public or private research centers.
L'archive ouverte pluridisciplinaire HAL, est destinée au dépôt et à la diffusion de documents scientifiques de niveau recherche, publiés ou non, émanant des établissements d'enseignement et de recherche français ou étrangers, des laboratoires publics ou privés. 
Modern Physics Letters A

Vol. 33, No. 22 (2018) 1850119 (20 pages)

(C) World Scientific Publishing Company

DOI: $10.1142 /$ S0217732318501195

\title{
Light-front and conformal field theories in two dimensions
}

\author{
Pierre Grangé* \\ Laboratoire Univers et Particules, Université de Montpellier, CNRS/IN2P3, \\ Place E. Bataillon, F-34095 Montpellier Cedex 05, France \\ pierre.grange@umontpellier.fr \\ Ernst Werner \\ Theoretische Physik, Universität Regensburg, Universitätstrasse 31, \\ D-93053 Regensburg, Germany \\ Ernst.Werner@physik.uni-regensburg.de
}

\author{
Received 19 January 2018 \\ Revised 21 April 2018 \\ Accepted 31 May 2018 \\ Published 10 July 2018
}

\begin{abstract}
Light-front (LF) quantization of massless fields in two spacetime dimensions is a long-standing and much debated problem. Even though the classical wave-equation is well-documented for almost two centuries, either as problems with initial values in spacetime variables or with initial data on characteristics in light-cone variables, the way to a consistent quantization in both types of frames is still a puzzle in many respects. This is in contrast to the most successful Conformal Field Theoretic (CFT) approach in terms of complex variables $z, \bar{z}$ pioneered by Belavin, Polyakov and Zamolodchikov in the ' $80 \mathrm{~s}$. It is shown here that the $2 \mathrm{D}$-massless canonical quantization in both reference frames is completely consistent provided that quantum fields are treated as OperatorValued Distributions (OPVD) with Partition of Unity (PU) test functions. We recall first that classical fields have to be considered as distributions (e.g. generalized functions in the Russian literature). Then, a necessary condition on the PU test function follows from the required matching of the classical solutions of the massless differential equations in both types of reference frame. Next we use a mathematical formulation for OPVD, developed in the recent past. Specifically, smooth $\mathcal{C}^{\infty}$ fields are introduced through the convolution operation in the distributional context. Due to the specific behavior of the Fourier-transform of the initial test function, this convolution transform has a well-defined integral in the dual space, whatever the initial choice of the reference frame. The relation to the conformal fields method follows immediately from the transition to Euclidean time and leads directly to explicit calculations of a few correlation functions of the scalar field and its energy-momentum tensor. The LF derivation of
\end{abstract}

\footnotetext{
* Corresponding author
} 


\begin{abstract}
the Virasoro algebra is then obtained from the $z$ and $\bar{z}$ expansions of the canonical fields as distributional Laplace-transform in these variables. Finally, the popular and problematic Discretized Light Cone Quantization (DLCQ) method is scrutinized with respect to its zero mode and ultraviolet content as encompassed in the continuum OPVD formulation.
\end{abstract}

Keywords: 2D spacetime; light-cone; conformal symmetry.

PACS Nos.: 03.70+k, 11.10.Kk, 11.10.Ef, 11.25.Hf

\title{
1. Introduction
}

As emphasized by $\operatorname{Dirac}^{1,2}$ in the light-front $(\mathrm{LF})$ spacetime and field variables, there are only three dynamical Poincaré generators and all three boost operators are kinematical. The vacuum structure is drastically simplified, but this apparent simplicity raises serious questions concerning the description of the symmetry breaking and vacuum condensation in the LF theory ( $\mathrm{LFT}$ ). It took a long time to realize that in LFT, the phase transition is driven by a coupling between two distinct parts of the field operator ${ }^{3}$ : the static zero mode and quantum fluctuations of the particle field. The zero mode carries essential non-perturbative-informations which in Equal Time formulation (EQT) are present in the nontrivial vacuum structure. On the other hand, the presence of constrained variables requires, in principle, specific and elaborate quantization methods for constrained systems.,$\frac{4,5}{}$ These additional difficulties with respect to the CFT formulation ${ }^{6-9}$ hindered a wider acceptance of the LFT approach, despite its great potential. $\underline{10-16}$ To clearly set up the logic of our argumentation leading to the conclusion of full consistency between EQT and LFT quantizations of two-dimensional massless fields, one has to first come back to the properties of the original classical wave-equation in its two different forms. The presentation is organized as follows. In Sec. 2 , we review 2D-massless instantaneous quantization as a Cauchy Problem and as a Characteristic Initial Value Problem (CIVP); in this case, we elaborate further on the necessary conditions making possible quantization on a single light-front. The final expression for the scalar massless quantum field is given, which shows explicitly its validity, both in ET and LF reference frames. In the first part of Sec. 3, the relation to the conformal fields method is obtained from the transition to Euclidean time in the scalar massless quantum field. Explicit calculations of a few correlation functions of this scalar field and its energy-momentum tensor are in full agreement with usual CFT results. Then, the LF derivation of the Virasoro algebra is obtained from the $z$ and $\bar{z}$ expansions of the canonical fields as distributional Laplace-transform in these variables. The second part of Sec. $\underline{3}$ elaborates on the DLCQ method with respect to its zero mode and ultraviolet contents as encompassed in the continuum OPVD formulation. Finally, some general comments and conclusions in relation to the earlier works on 2D-massless bosonic and fermionic fields are given in the last section. 


\section{Solutions of the 2D-Massless Wave Equation and Instantaneous Quantizations}

\subsection{The Cauchy problem and its solution as a classical tempered distribution in spacetime variables}

Consider a two-dimensional Minkowski flat space $\mathcal{M}$ with coordinates $x=\left(x^{0}, x^{1}\right)$ (with a pseudo-metric $g_{\mu, \nu}=\operatorname{diag}\{1,-1\}$, with $\{\mu, \nu\} \in\{0,1\} ; \square=\partial^{\mu} g_{\mu \nu} \partial^{\nu}$ and $\langle\langle x, y\rangle\rangle=x^{\mu} g_{\mu \nu} y^{\nu}$ the associated bilinear form). To the classical field-distribution $\phi\left(x^{0}, x^{1}\right)$ is then associated a linear functional $\Phi[\rho]$ built on test functions on Schwartz spaces $\mathcal{S}^{17,18}$ such that $\rho:(\mathbf{I R} \times \mathbf{I R}) \ni\left(x^{0}, x^{1}\right) \mapsto \rho\left(x^{0}, x^{1}\right) \in \mathcal{S}\left(\overline{\mathbf{R}}^{2}\right)$ (the completed topological tensor product $\left.\mathcal{S}(\mathbf{R}) \hat{\otimes} \mathcal{S}(\mathbf{R})^{19}\right)$. It is important to note that, due to separate reflexion symmetry of the test function $\rho$ in the variables $x^{0}$ and $x^{1}$, the translation-convolution product can still be written as an integral in Fourier-space variables with the proper pseudo-metric bilinear form, ${ }^{20,21}$ that is

$$
(\Phi * \rho)\left(x^{0}, x^{1}\right)=\left\langle\tilde{\Phi}, e^{(-i\langle\langle\ldots, x\rangle\rangle)} \rho\right\rangle=\int \frac{d p_{0} d p_{1}}{(2 \pi)^{2}} e^{-i\langle\langle p, x\rangle\rangle} \tilde{\phi}\left(p^{0}, p_{1}\right) \tilde{\rho}\left(p_{0}^{2}, p_{1}^{2}\right),
$$

where $\tilde{\phi}\left(p^{0}, p_{1}\right)$ (resp. $\left.\tilde{\rho}\left(p_{0}^{2}, p_{1}^{2}\right)\right)$, is the Fourier-space transform of $\phi\left(x^{0}, x^{1}\right)$ (resp. of $\left.\rho\left(x^{0}, x^{1}\right)\right)$. Hereafter, $\boldsymbol{\Phi}\left(x^{0}, x^{1}\right)$ will stand for $(\Phi * \rho)\left(x^{0}, x^{1}\right)$ and the starting covariant action is

$$
I=\int_{\mathcal{M}} d^{2} x \mathcal{L}=\frac{1}{2} \int_{\mathcal{M}} d^{2} x \square \mathbf{\Phi}\left(x^{0}, x^{1}\right) .
$$

The principle of least action yields

$$
\delta I=\int_{\mathcal{M}} d^{2} x\left[\frac{\partial \mathcal{L}}{\partial \boldsymbol{\Phi}}-\partial_{\mu} \frac{\partial \mathcal{L}}{\partial\left(\partial_{\mu} \boldsymbol{\Phi}\right)}\right] \delta \phi+\int_{\partial \mathcal{M}} d \sigma_{\mu} \frac{\partial \mathcal{L}}{\partial\left(\partial_{\mu} \boldsymbol{\Phi}\right)} \delta \boldsymbol{\Phi}=0 .
$$

From the convolution construction, $\boldsymbol{\Phi}\left(x^{0}, x^{1}\right)$ is $\mathbb{C}^{\infty}$, belongs to $\mathcal{S}\left(\overline{\mathbf{R}}^{2}\right)$, and the surface term in $\delta I$ vanishes surely, resulting in the Euler-Lagrange (EL) equations. The wave-equation for the classical convoluted distribution in spacetime variables is obtained ${ }_{-}^{\mathrm{a}}$ from the hyperbolic partial differential equation (HPDE)

$$
\square \boldsymbol{\Phi}\left(x^{0}, x^{1}\right)=\left[\partial_{x^{0}}^{2}-\partial_{x^{1}}^{2}\right] \boldsymbol{\Phi}\left(x^{0}, x^{1}\right)=0 .
$$

With the notation $\mathbb{D}_{x^{1}}=\partial_{x^{1}}^{2}$, this is equivalent to the system

$$
\left\{\begin{array}{l}
\partial_{x^{0}} \mathbf{U}=\mathbf{V} \\
\partial_{x^{0}} \mathbf{V}=\mathbb{D}_{x^{1}} \mathbf{U}
\end{array}\right.
$$

A solution of the Cauchy problem in the sense of convolution of tempered distributions is a vector $\mathbf{W}=\left(\begin{array}{l}\mathbf{U} \\ \mathbf{V}\end{array}\right)$ such that

$$
\partial_{x^{0}} \mathbf{W}+A\left(D_{x^{1}}\right) \mathbf{W}=0 \quad \text { with } A\left(D_{x}\right)=-\left(\begin{array}{cc}
0 & \mathbb{1} \\
\mathbb{D}_{x} & 0
\end{array}\right)
$$

and with $\left.\mathbf{W}\right|_{x^{0}=0}=\left(\begin{array}{c}\mathbf{f}\left(x^{1}\right) \\ \mathbf{g}\left(x^{1}\right)\end{array}\right)$, with $\left(\mathbf{f}\left(x^{1}\right), \mathbf{g}\left(x^{1}\right)\right)$ the initial convoluted data $\in \mathcal{S}(\mathbf{R})$.

${ }^{a}\left\{x^{0}, x^{1}\right\}$ are chosen dimensionless, implying the presence of an arbitrary scale $\Lambda$ such that $x^{0}=$ $\Lambda(c t)$, with $c$ the propagation speed, $t$ the time variable and $x^{1}=\Lambda x$, with $x^{0}$ the space variable. 
An invariant elementary solution $\boldsymbol{\Delta}(x)$ of Eq. (1) is built on the anti-symmetric tempered distribution

$$
\Delta(x)=-\frac{1}{2} \operatorname{sign}\left(x^{0}\right) H\left(x^{2}\right),
$$

where $H\left(x^{2}\right)$ is the Heaviside distribution of argument $x^{2}=\left(x^{0}\right)^{2}-\left(x^{1}\right)^{2} \cdot H\left(x^{2}\right)$ vanishes for $x^{2}<0$, that is outside the light-cone. The full solution of Eq. (1) can be expressed through its initial values $\mathbf{f}$ and $\mathbf{g}$ and reads

$$
\boldsymbol{\Phi}\left(x^{0}, x^{1}\right)=\int_{-\infty}^{\infty} d y^{1}\left[\mathbf{f}\left(y^{1}\right) \partial_{y^{0}} \boldsymbol{\Delta}(x-y)-\mathbf{g}\left(y^{1}\right) \boldsymbol{\Delta}(x-y)\right],
$$

which, if evaluated with the elementary solution $\boldsymbol{\Delta}(x)$ built on Eq. (4), is nothing else than D'Alembert's (1717-1783) solution ${ }^{\mathrm{b}}$

$$
\mathbf{\Phi}\left(x^{0}, x^{1}\right)=\frac{1}{2}\left[\mathbf{f}\left(x^{1}-x^{0}\right)+\mathbf{f}\left(x^{1}+x^{0}\right)\right]+\frac{1}{2} \int_{x^{1}-x^{0}}^{x^{1}+x^{0}} d y \mathbf{g}(y) .
$$

In terms of the Fourier transform $\tilde{f}$ (resp. $\tilde{g}, \tilde{\rho}$ ) of the initial distributions $f$ (resp. $g, \rho$ ), the convolution product $\mathbf{f}(u)$ takes the form

$$
\mathbf{f}(u)=\int_{-\infty}^{\infty} d z f(z) \rho(u-z)=\frac{1}{2 \pi} \int_{-\infty}^{\infty} d p e^{\imath p u} \tilde{f}(p) \tilde{\rho}\left(p^{2}\right)
$$

and gives

$$
\begin{aligned}
\mathbf{f}\left(x^{1}-x^{0}\right)+\mathbf{f}\left(x^{1}+x^{0}\right) & =\frac{1}{\pi} \int_{-\infty}^{\infty} d p e^{\imath p x^{1}} \cos \left(|p| x^{0}\right) \tilde{f}(p) \tilde{\rho}\left(p^{2}\right) \\
& \equiv \frac{1}{2 \pi} \int_{-\infty}^{\infty} d p\left[e^{\imath\left(p x^{1}-|p| x^{0}\right)}+e^{\imath\left(p x^{1}+|p| x^{0}\right)}\right] \tilde{f}(p) \tilde{\rho}\left(p^{2}\right)
\end{aligned}
$$

In the same way, the second contribution to D'Alembert's solution, with $u=x^{1}-x^{0}$ and $v=x^{1}+x^{0}$, can be written as

$$
\begin{aligned}
\int_{u}^{v} d y \mathbf{g}(y) & =\frac{1}{2 \pi} \int_{u}^{v} d y \int_{-\infty}^{\infty} d p e^{\imath p y} \tilde{g}(p) \tilde{\rho}\left(p^{2}\right) \\
& =\frac{1}{2 \imath \pi} \int_{-\infty}^{\infty} \frac{d p}{p}\left(e^{\imath p v}-e^{\imath p u}\right) \tilde{g}(p) \tilde{\rho}\left(p^{2}\right) \\
& =\frac{1}{2 \imath \pi} \int_{-\infty}^{\infty} \frac{d p}{|p|}\left[e^{\imath\left(p x^{1}+|p| x^{0}\right)}-e^{\imath\left(p x^{1}-|p| x^{0}\right)}\right] \tilde{g}(p) \tilde{\rho}\left(p^{2}\right) .
\end{aligned}
$$

Regrouping terms in Eq. (6) finally gives

$$
\boldsymbol{\Phi}\left(x^{0}, x^{1}\right)=\frac{1}{4 \pi} \int_{-\infty}^{\infty} \frac{d p}{|p|}\left[e^{-\imath\left(|p| x^{0}-p x^{1}\right)} \chi_{+}(p)+e^{\imath\left(|p| x^{0}+p x^{1}\right)} \chi_{-}(p)\right] \tilde{\rho}\left(p^{2}\right),
$$

\footnotetext{
${ }^{\mathrm{b}}$ It should not come as a surprise, for the convolution leads to smooth $\mathbb{C}^{\infty}$ and fast decreasing functions at large distances, in conformity with D'Alembert's assumptions.
} 
with

$$
\chi_{ \pm}(p)=|p| \tilde{f}(p) \pm \imath \tilde{g}(p)
$$

in agreement with the alternative expression deduced from the form

$$
\boldsymbol{\Phi}\left(x^{0}, x^{1}\right)=\frac{1}{2 \pi} \int d^{2} p \delta\left(p_{0}^{2}-p_{1}^{2}\right) \chi\left(p_{0}, p_{1}\right) e^{-\imath\left(p_{0} x^{0}-p_{1} x^{1}\right)} \tilde{\rho}\left(p_{0}^{2}, p_{1}^{2}\right),
$$

with $\chi\left( \pm\left|p_{1}\right|, p_{1}\right)=\chi_{ \pm}\left(p_{1}\right)$.

Following the usual approach of canonical quantization ${ }^{21}$ (Sec. 2.4) the zero mass scalar quantum field OPVD $\hat{\Phi}\left(x^{0}, x^{1}\right)$ proceeds from Eq. (7) via the correspondence in terms of creation and annihilation operators $\left\{\chi_{-}(p) \curvearrowright a^{\dagger}(p), \chi_{+}(p) \curvearrowright a(p)\right\}$, with commutator algebra $\left[a(p), a^{+}(q)\right]=4 \pi p \delta(p-q)$ and a vacuum $|0\rangle$ such that $a(p)|0\rangle=0 \forall p$. That is

$$
\hat{\Phi}\left(x^{0}, x^{1}\right)=\frac{1}{4 \pi} \int_{0}^{\infty} \frac{d p}{p}\left[a(p) e^{-\imath p\left(x^{0}-x^{1}\right)}+a^{\dagger}(p) e^{\imath p\left(x^{0}+x^{1}\right)}\right] \tilde{\rho}\left(p^{2}\right) .
$$

Then, one easily evaluates the commutator of two free scalar OPVD to

$$
[\hat{\Phi}(x), \hat{\Phi}(\mathbf{0})]=-\frac{\imath}{\pi} \int_{0}^{\infty} \frac{d p}{p} \sin \left(p x^{0}\right) \cos \left(p x^{1}\right) \tilde{\rho}^{2}\left(p^{2}\right) .
$$

This integral is finite without the test function and it is important to understand the limiting procedure leading to $\tilde{\rho}^{2}\left(p^{2}\right) \equiv \tilde{\rho}\left(p^{2}\right)=1$. At first sight, there is a high degree of arbitrariness in the choice of $\tilde{\rho}$ which is seemingly fatal to the uniqueness of the resulting field theory. However, it is the mathematically well-established nature of Minkowskian and Euclidean dual spaces as paracompact entities equipped with PU test functions which permits this uniqueness requirement, for the product of two PUs is a PU and any further convolution beyond the first one becomes redundant. The simultaneous treatment of ultraviolet and infrared singularities follows. It yields then $\mathbb{C}^{\infty}$ quantities with simple convoluted spacetime integrals, for the inverse Fourier transform of the PU test function in the limiting procedure where $\tilde{\rho}^{2}\left(p^{2}\right) \equiv \tilde{\rho}\left(p^{2}\right)=1$ is just Dirac's $\delta$-distribution. These aspects are exposed at some length in Refs. 22 and 24 and we only give here a short account to clarify the presentation.

A PU can be built up from a basic super regular function $u(X)$ such that

$$
u(X)+u(h-X)=1 \quad \text { for any } X \in[0, h],
$$

where $h$ is a positive real number, and take

$$
\beta_{i}(X) \equiv u(|X-i h|) \quad \text { for }|X-i h|<h .
$$

Then a PU is obtained as

$$
f_{\mathrm{PU}}(X)=\sum_{i=1}^{N} \beta_{i}(X)
$$


with the following properties

$$
f_{\mathrm{PU}}(X)= \begin{cases}u(h-X), & \text { for } X \in[0, h], \\ 1, & \text { for } X \in[h, N h], \\ u(X-N h), & \text { for } X \in[N h,(N+1) h] .\end{cases}
$$

The covering of the space (here a line segment) is therefore accomplished by the sum over subspaces $\Omega_{i}$ to which the $\beta_{i}$ are subordinates. It is important to note that the super regular properties as well as relation (11) for the functions $\beta_{i}$ are preserved when $h$ depends on $X$. It is non-restrictive and convenient to parametrize $h(X)$ as follows:

$$
h(X)=\eta^{2} X g_{\alpha}(X)+(\alpha-1)
$$

where $\eta$ and $\alpha$ are positive parameters. With this choice of $h(X)$, it is sufficient to consider only two functions $\beta_{i}$, with $i=1,2$, to build up the simplest PU. $\beta_{1}$ and $\beta_{2}$ will vanish, respectively, with all their derivatives when $X=h(X)$ and when $X=2 h(X)$. We demand that when $\alpha \rightarrow 1^{-}$, the sum of the two $\beta$ s cover the whole integration domain in $X$. Further technical details are given in the Appendices.

In this way, $\tilde{\rho}^{2}\left(p^{2}\right)$ in Eq. (10) can be taken to unity and the usual result follows:

$$
[\hat{\Phi}(x), \hat{\Phi}(\mathbf{0})]=\imath \Delta(x) .
$$

\subsection{The characteristic initial value problem (CIVP) and its solution as a classical tempered distributions in light-cone variables}

Our starting point is to introduce LC variables $x^{ \pm}=x^{0} \pm x^{1}$ that modify the initial Klein-Gordon (KG) equation to

$$
\partial_{+} \partial_{-} \Phi\left(x^{+}, x^{-}\right)=0 .
$$

The light-like hyperplanes $x^{ \pm}=$const. are called the characteristics of this hyperbolic partial-differential equation. The CIVP is defined as follows. Determine a solution $\Phi\left(x^{+}, x^{-}\right)$with initial data on characteristic surfaces

$$
\Phi\left(x^{+}, x_{0}^{-}\right)=\mathfrak{f}\left(x^{+}\right), \quad \Phi\left(x_{0}^{+}, x^{-}\right)=\mathfrak{g}\left(x^{-}\right)
$$

and the continuity condition

$$
\Phi\left(x_{0}^{+}, x_{0}^{-}\right)=\mathfrak{f}\left(x_{0}^{+}\right)=\mathfrak{g}\left(x_{0}^{-}\right) .
$$

Following Neville and Rohrlich, ${ }^{12}$ the relation of the CIVP to D'Alembert's solution of the Cauchy problem in Eq. (6) can be obtained via Gauss' theorem applied to the divergence-free expression

$$
F_{\mu}(x, y)=\boldsymbol{\Delta}(x-y) \frac{\partial}{\partial y^{\mu}} \boldsymbol{\Phi}(y)-\boldsymbol{\Phi}(y) \frac{\partial}{\partial y^{\mu}} \boldsymbol{\Delta}(x-y),
$$


for both $\boldsymbol{\Phi}$ and $\boldsymbol{\Delta}$ satisfy the KG equation. Integrating (19) over the simplex $A B C$, where on $A B: x^{0}=0$, on $B C: x^{-}=x_{0}^{-}$and on $C A: x^{+}=x_{0}^{+}$, yields by Gauss' theorem

$$
\left(\int_{A B}+\int_{B C}+\int_{C A}\right) d \sigma(y) n_{\mu} F^{\mu}(x, y)=0,
$$

where $d \sigma$ and $n_{\mu}$ are the appropriate surface element and unit vectors. The first term $\int_{A B}$ just corresponds to (5), that is the solution (6) of the Cauchy problem, whereas the two other terms give the expression for the solution of CIVP for the $\mathrm{KG}$ equation in terms of the initial data of (17). For zero mass, a very simple result is obtained ${ }^{23}$

$$
\begin{aligned}
\boldsymbol{\Phi}\left(x^{0}, x^{1}\right) & =\frac{1}{2}\left[\mathfrak{f}\left(x_{0}^{+}\right)+\mathfrak{g}\left(x_{0}^{-}\right)\right]+\frac{1}{2} \int_{x_{0}^{-}}^{x^{-}} d y^{-} \partial_{y}^{+} \mathfrak{g}\left(y^{-}\right)+\frac{1}{2} \int_{x_{0}^{+}}^{x^{+}} d y^{+} \partial_{y}^{-} \mathfrak{f}\left(y^{+}\right) \\
& =\mathfrak{f}\left(x^{+}\right)+\mathfrak{g}\left(x^{-}\right)-\frac{1}{2}\left[\mathfrak{f}\left(x_{0}^{+}\right)+\mathfrak{g}\left(x_{0}^{-}\right)\right] \equiv \mathfrak{F}\left(x^{+}\right)+\mathfrak{G}\left(x^{-}\right)
\end{aligned}
$$

It is always feasible to pick up the initial data functions giving a null value for the continuity condition (18). With the respective notations $\mathfrak{F}\left(x^{+}\right)$and $\mathfrak{G}\left(x^{-}\right)$for these initial data, (21) reduces to a consistency relation between the solution of the CIVP and that of the Cauchy problem

$$
\mathfrak{F}\left(x^{+}\right)+\mathfrak{G}\left(x^{-}\right)=\frac{1}{2}\left[\mathbf{f}\left(-x^{-}\right)+\mathbf{f}\left(x^{+}\right)\right]+\frac{1}{2} \int_{-x^{-}}^{x^{+}} d y \mathbf{g}(y) .
$$

Taking (22) and its derivative with respect to $x^{0}, \frac{\partial}{\partial_{x^{0}}}=\frac{\partial}{\partial_{x^{+}}}+\frac{\partial}{\partial_{x^{-}}}$, at $x^{0}=0$ result in two equations

$$
\mathbf{f}\left(x^{1}\right)=\mathfrak{F}\left(x^{1}\right)+\mathfrak{G}\left(-x^{1}\right),
$$

and

$$
\begin{aligned}
\mathbf{g}\left(x^{1}\right) & =\frac{\partial}{\partial_{x^{0}}}\left[\mathfrak{F}\left(x^{+}\right)+\mathfrak{G}\left(x^{-}\right)\right]_{x^{0}=0} \\
& =\left.\frac{\partial}{\partial_{x^{+}}} \mathfrak{F}\left(x^{+}\right)\right|_{x^{+}=x^{1}}+\left.\frac{\partial}{\partial_{x^{-}}} \mathfrak{G}\left(x^{-}\right)\right|_{x^{-}=-x^{1}} .
\end{aligned}
$$

To avoid unnecessary lengthy formulae, we can take $x_{0}^{+}=x_{0}^{-}=\mathbf{f}(0)=0$. Then, it is straightforward to get

$$
\begin{aligned}
& \mathfrak{F}\left(x^{+}\right)=\frac{1}{2}\left[\mathbf{f}\left(x^{+}\right)+\int_{0}^{x^{+}} d y \mathbf{g}(y)\right], \\
& \mathfrak{G}\left(x^{-}\right)=\frac{1}{2}\left[\mathbf{f}\left(-x^{-}\right)-\int_{0}^{-x^{-}} d y \mathbf{g}(y)\right] .
\end{aligned}
$$

This establishes the complete consistency of the classical solutions of the zero-mass scalar wave propagation as a Cauchy problem and as a CIVP. It is also true for a 
nonzero mass $m$ as shown in Ref. 23, but more elaborate, for the Riemann function $J_{0}\left(m \sqrt{x^{+} x^{-}}\right)$mixes the propagation on the sections $B C$ and $C A$ of the simplex $A B C$.

Thereby, the Hamiltonian formulation of canonical quantization is generic, in so far as the appearance of (primary) constraints from the singular LC-Lagrangian is not of physical significance. $\frac{5}{}$ In effect, it amounts to requiring equivalence between EL and Hamiltonian equations (see below).

\subsection{Lessons from a careful analysis of the massless limit of the massive LFT scalar solutions}

Our light-front notations for a nonzero mass $m$ are as follows:

$$
\begin{gathered}
x^{\mu}=\left(x^{+}, x^{-}\right), \quad p^{\mu}=\left(p^{+}, p^{-}\right), \quad \partial_{ \pm}=\frac{\partial}{\partial x^{ \pm}}, \\
\hat{p} \cdot x=\frac{1}{2} p^{+} x^{-}+\frac{1}{2} \hat{p}^{-} x^{+}, \quad \hat{p}^{-}=\frac{m^{2}}{p^{+}} \\
p^{0}=p^{+}+\hat{p}^{-}, \quad p^{1}=p^{+}-\hat{p}^{-}, \\
\tilde{\rho}\left[\left(p^{0}\right)^{2},\left(p^{1}\right)^{2}\right]=\tilde{\rho}\left[\left(p^{+}+\hat{p}^{-}\right)^{2},\left(p^{+}-\hat{p}^{-}\right)^{2}\right] .
\end{gathered}
$$

The covariant LF Lagrangian and the KG equation take the forms

$$
\mathcal{L}=2 \partial_{+} \boldsymbol{\Phi} \partial_{-} \boldsymbol{\Phi}-\frac{1}{2} m^{2} \boldsymbol{\Phi}^{2}, \quad\left(4 \partial_{+} \partial_{-}+m^{2}\right) \boldsymbol{\Phi}=0 .
$$

The corresponding momentum and field time derivative are

$$
\pi(x)=2 \partial_{-} \boldsymbol{\Phi}(x), \quad \theta(x)=2 \partial_{+} \boldsymbol{\Phi}(x) .
$$

The components of the canonical energy-momentum tensor

$$
T^{\mu \nu}(x)=\frac{\partial \mathcal{L}}{\partial_{\mu} \boldsymbol{\Phi}(x)} \partial^{\nu} \boldsymbol{\Phi}(x)-g^{\mu \nu} \mathcal{L},
$$

corresponding to the Lagrangian (28), are

$$
\begin{aligned}
& T^{++}(x)=4 \partial_{-} \boldsymbol{\Phi}(x) \partial_{-} \boldsymbol{\Phi}(x), \\
& T^{--}(x)=4 \partial_{+} \boldsymbol{\Phi}(x) \partial_{+} \boldsymbol{\Phi}(x), \\
& T^{+-}(x)=m^{2} \boldsymbol{\Phi}^{2}(x)=T^{-+}(x) .
\end{aligned}
$$

The quantum solution of the field equation (28) takes the form ${ }^{20,21}$

$$
\hat{\mathbf{\Phi}}(x)=\int_{0}^{\infty} \frac{d p^{+}}{4 \pi p^{+}}\left[a\left(p^{+}\right) e^{-\frac{i}{2} \hat{p} \cdot x}+a^{\dagger}\left(p^{+}\right) e^{\frac{i}{2} \hat{p} \cdot x}\right] \tilde{\rho}\left[\left(p^{+}+\hat{p}^{-}\right)^{2},\left(p^{+}-\hat{p}^{-}\right)^{2}\right],
$$

with commutator algebra

$$
\left[a(p), a^{+}(q)\right]=4 \pi p \delta(p-q)
$$


and an LF vacuum $|0\rangle$ such that $a(p)|0\rangle=0 \forall p$. To simplify subsequent writings, we adopt the shorthand notation $\tilde{\rho}\left[\left(p^{+}+\hat{p}^{-}\right)^{2},\left(p^{+}-\hat{p}^{-}\right)^{2}\right] \equiv \widetilde{\rho p}\left(p^{+}, \frac{m^{2}}{p^{+}}\right)$with $\left.\widetilde{\rho p}\left(p^{+}, \frac{m^{2}}{p^{+}}\right)\right|_{m=0}=\tilde{\rho}\left[\left(p^{+}\right)^{2},\left(p^{+}\right)^{2}\right) \equiv \tilde{\rho}\left[\left(p^{+}\right)^{2}\right]$. With the fields as OPVD, the presence of a PU test function such that $\forall n \geq 0 \lim _{p+\rightarrow 0^{+}} \frac{d^{n}}{d\left(p^{+}\right)^{n}} \tilde{\rho}\left[\left(p^{+}\right)^{2}\right] \rightarrow 0$ faster than any inverse power of $p^{+}$(cf. comments after Eq. (9), Appendix A and the IR extension procedure ${ }^{21}$ for a mathematically complete account) ensures the necessary condition $\left.\frac{\tilde{\rho}\left[\left(p^{+}\right)^{2}\right]}{p^{+}}\right|_{p^{+}=0}=0$ for $(34)$ to be well-defined in LC variables. ${ }^{23}$ From (29) and (34) we directly find

$$
\begin{aligned}
& \theta(x)=-i \int_{0}^{\infty} \frac{d p^{+}}{4 \pi p^{+}} \frac{m^{2}}{p^{+}}\left[a\left(p^{+}\right) e^{-\frac{i}{2} \hat{p} \cdot x}-a^{\dagger}\left(p^{+}\right) e^{\frac{i}{2} \hat{p} \cdot x}\right] \widetilde{\rho p}\left(p^{+}, \frac{m^{2}}{p^{+}}\right), \\
& \pi(x)=-i \int_{0}^{+\infty} \frac{d p^{+}}{4 \pi}\left[a\left(p^{+}\right) e^{-\frac{i}{2} \hat{p} \cdot x}-a^{\dagger}\left(p^{+}\right) e^{\frac{i}{2} \hat{p} \cdot x}\right] \widetilde{\rho p}\left(p^{+}, \frac{m^{2}}{p^{+}}\right) .
\end{aligned}
$$

The LF Hamiltonian and the momentum operator are

$$
\begin{aligned}
& P^{-}=\frac{1}{2} \int_{-\infty}^{+\infty} d x^{-} T^{+-}(x)=m^{2} \int_{0}^{\infty} \frac{d p^{+}}{4 \pi p^{+}} \frac{1}{p^{+}} a^{\dagger}\left(p^{+}\right) a\left(p^{+}\right) \widetilde{\rho p}^{2}\left(p^{+}, \frac{m^{2}}{p^{+}}\right), \\
& P^{+}=\frac{1}{2} \int_{-\infty}^{+\infty} d x^{-} T^{++}(x)=\int_{0}^{\infty} \frac{d p^{+}}{4 \pi} a^{\dagger}\left(p^{+}\right) a\left(p^{+}\right) \widetilde{\rho p}^{2}\left(p^{+}, \frac{m^{2}}{p^{+}}\right) .
\end{aligned}
$$

Since, besides the test function, the mass dependence resides only in the planewave factor, ${ }_{2}^{\text {c }}$ the massless limit of the massive solution $(34)$ yields $\hat{\mathfrak{G}}\left(x^{-}\right)$in a straightforward way

$$
\hat{\mathfrak{G}}\left(x^{-}\right)=\int_{0}^{\infty} \frac{d p^{+}}{4 \pi p^{+}}\left[a\left(p^{+}\right) e^{-\frac{i}{2} p^{+} x^{-}}+a^{\dagger}\left(p^{+}\right) e^{\frac{i}{2} p^{+} x^{-}}\right] \tilde{\rho}\left[\left(p^{+}\right)^{2}\right] .
$$

But where is the second piece $\hat{\mathfrak{F}}\left(x^{+}\right)$? From the analysis of the CIVP, it is also contained in (34). To find it, make the change of variable $p^{+} \mapsto \frac{m^{2}}{p^{-}}$in (34). This is a legitimate step, for the integral is well defined in the presence of a test function. It gives

$$
\hat{\mathfrak{F}}\left(x^{+}\right)=\int_{0}^{\infty} \frac{d p^{-}}{4 \pi p^{-}}\left[\lim _{m \rightarrow 0} a\left(\frac{m^{2}}{p^{-}}\right) e^{-\frac{i}{2} p^{-} x^{+}}+\text {H.c. }\right] \tilde{\rho}\left[\left(p^{-}\right)^{2}\right] .
$$

Next, calculate the Fock commutators in terms of the new variables:

$$
\begin{aligned}
\lim _{m \rightarrow 0}\left[a\left(\frac{m^{2}}{p^{-}}\right), a^{\dagger}\left(\frac{m^{2}}{q^{-}}\right)\right] & =\lim _{m \rightarrow 0} 4 \pi \frac{m^{2}}{p^{-}} \delta\left(\frac{m^{2}}{p^{-}}-\frac{m^{2}}{q^{-}}\right) \\
& =4 \pi p^{-} \delta\left(p^{-}-q^{-}\right) .
\end{aligned}
$$

${ }^{\mathrm{c}}$ It had been realized long ago that the measure in the LF momentum integrals is massindependent, $\frac{15}{2}$ at variance to the space-like form of the theory. 
Since the right-hand side does not depend on mass and hence survives the massless limit, this should be true for the left-hand side as well. So

$$
\lim _{m \rightarrow 0} a\left(\frac{m^{2}}{k^{-}}\right)\left(\text {viz. } a^{\dagger}\left(\frac{m^{2}}{k^{-}}\right)\right) \equiv \tilde{a}\left(p^{-}\right)\left(\text {viz. } \tilde{a}^{\dagger}\left(p^{-}\right)\right),
$$

exist as annihilation and creation operators, with the properties

$$
\left[\tilde{a}\left(p^{-}\right), \tilde{a}^{\dagger}\left(q^{-}\right)\right]=4 \pi p^{-} \delta\left(p^{-}-q^{-}\right), \quad\left[a\left(p^{+}\right), \tilde{a}^{\dagger}\left(q^{-}\right)\right]=0 .
$$

Performing the massless limit in the expression (39) and in $\pi(x), \theta(x)$ from (29), we find

$$
\begin{gathered}
\hat{\mathfrak{F}}\left(x^{+}\right)=\int_{0}^{\infty} \frac{d p^{-}}{4 \pi p^{-}}\left[\tilde{a}\left(p^{-}\right) e^{-\frac{i}{2} p^{-} x^{+}}+\tilde{a}^{\dagger}\left(p^{-}\right) e^{\frac{i}{2} p^{-} x^{+}}\right] \tilde{\rho}\left[\left(p^{-}\right)^{2}\right], \\
\theta\left(x^{+}\right)=-\frac{i}{4 \pi} \int_{0}^{\infty} d p^{-}\left[\tilde{a}\left(p^{-}\right) e^{-\frac{i}{2} p^{-} x^{+}}-\text {H.c. }\right] \tilde{\rho}\left[\left(p^{-}\right)^{2}\right]
\end{gathered}
$$

and

$$
\pi\left(x^{-}\right)=-\frac{i}{4 \pi} \int_{0}^{\infty} d p^{+} \tilde{\rho}\left[\left(p^{+}\right)^{2}\right]\left[a\left(p^{+}\right) e^{-\frac{i}{2} p^{+} x^{-}}-\text {H.c. }\right] .
$$

The change of variables in (39) had to be performed for $\theta(x)$ to make it compatible with the equation of motion $\partial_{+} \partial_{-} \boldsymbol{\Phi}(x)=0$, that is to depend on $x^{+}$only. The basic commutators, following from (35) and (42), are easily obtained in the same way as that of Eq. (10) to give

$$
\begin{aligned}
{\left[\hat{\mathfrak{G}}\left(x^{-}\right), \hat{\mathfrak{G}}\left(y^{-}\right)\right] } & =-\frac{i}{4} \operatorname{sign}\left(x^{-}-y^{-}\right), \\
{\left[\hat{\mathfrak{F}}\left(x^{+}\right), \hat{\mathfrak{F}}\left(y^{+}\right)\right] } & =-\frac{i}{4} \operatorname{sign}\left(x^{+}-y^{+}\right) .
\end{aligned}
$$

In this way, the second half of the solution of the wave equation has been recovered from the massive solution. Note also that the variables $p^{+}$and $p^{-}$in fact coincide. This is analogous to the SL case where we have $p^{0}=\left|p^{1}\right|$. In the LF case, we have directly $p^{-} \equiv p^{+}$since both are positive-definite.

As a consistency check, the two-point functions calculated from the massless field should coincide with the massless limit of the massive functions. This is indeed the case for $D_{1}^{(+)}(x-y)=\langle 0|\hat{\boldsymbol{\Phi}}(x) \pi(y)| 0\rangle$ and $D_{2}^{(+)}(x-y)=\langle 0|\hat{\boldsymbol{\Phi}}(x) \theta(y)| 0\rangle$. On the other hand, $D_{0}^{(+)}(x-y)=\langle 0|\hat{\boldsymbol{\Phi}}(x) \hat{\boldsymbol{\Phi}}(y)| 0\rangle$ calculated from the massless solution is ill-defined with $m$ carelessly set to zero from the start of the calculation. The rigorous treatment involves first the extension of singular distributions in $\frac{1}{\left(p^{+}\right)^{n}}$ with the test function $\tilde{\rho}\left[\left(p^{+}\right)^{2}\right]$ and then the calculation of Laplace transforms, as detailed in Ref. 21. $D_{0}^{(+)}(x-y)$ is known to behave ${ }^{7}$ as $\ln (|x-y|)$ (cf. footnote a).

It is instructive to study the massless limit of the LF momentum and Hamiltonian. One expects that these operators survive the massless limit, for a consistent quantum theory has to lead to the Heisenberg equations

$$
2 i \partial_{+} \hat{\mathfrak{F}}\left(x^{+}\right)=-\left[P^{-}, \hat{\mathfrak{F}}\left(x^{+}\right)\right], \quad 2 i \partial_{-} \hat{\mathfrak{G}}\left(x^{-}\right)=-\left[P^{+}, \hat{\mathfrak{G}}\left(x^{-}\right)\right] .
$$


The massless limit of the momentum operator (37) is straightforward, while the change of variables (39) is necessary for the Hamiltonian. Using the Fock commutators (42) in this case, the resultant operators

$$
\begin{aligned}
& P^{+}=\int_{0}^{\infty} \frac{d p^{+}}{4 \pi} a^{\dagger}\left(p^{+}\right) a\left(p^{+}\right) \tilde{\rho}^{2}\left[\left(p^{+}\right)^{2}\right], \\
& P^{-}=\int_{0}^{\infty} \frac{d p^{-}}{4 \pi} \tilde{a}^{\dagger}\left(p^{-}\right) \tilde{a}\left(p^{-}\right) \tilde{\rho}^{2}\left[\left(p^{-}\right)^{2}\right],
\end{aligned}
$$

are easily seen to generate the Heisenberg equations (48). ${ }^{\mathrm{d}}$ On the other hand, the massless limit of the LF Hamiltonian density based on the canonical form of $T^{+-}(x)$ in Eq. (33) would seem to vanish without the CIVP considerations leading to (42). The underlying conformal symmetry of the massless theory requires

$$
\operatorname{Tr} T^{\mu \nu}=T_{\mu}^{\mu}=0
$$

It is well known ${ }^{7}$ that the tracelessness of $T$ can be achieved by a modification of the canonical form of Eq. (30) leaving the action invariant.

\section{Conformal Symmetry Properties of the Massless LF Scalar Field in Two Dimensions}

\subsection{Conformal symmetry characterization from LFT analysis in the continuum}

CFT is formulated in terms of independent complex variables $\{z, \bar{z}\}$ with $z=\tau-\imath x^{1}$, $z^{*}=\tau+\imath x^{1}, \tau$ the Euclidean time and $\bar{z}=z^{*}$ on the real surface (the usual bidimensional Euclidean space). Then, the initial KG equation (16) takes the form

$$
\partial_{z} \partial_{\bar{z}} \phi(z, \bar{z})=0 \text {. }
$$

The generic scalar field solution in the continuum is $\phi(z, \bar{z})=\varphi(z)+\varphi(\bar{z})$ and classically we then have

$$
\begin{aligned}
\varphi(z) & =\int_{0}^{\infty} \frac{d p}{4 \pi p}\left[\chi(-p) e^{\frac{1}{2} p z}+\chi(p) e^{-\frac{1}{2} p z}\right] \tilde{\rho}\left(p^{2}\right) \\
& =I^{+}(z)+I^{-}(z) .
\end{aligned}
$$

With the change of variable $p=t \Lambda$, with $\Lambda$ an arbitrary positive scale, and expanding $\chi( \pm t \Lambda)$ in a Taylor series around $t=0, I^{\mp}(z)$ in Eq. (52) reads

$$
I^{\mp}(z)=\frac{1}{4 \pi} \sum_{n=0}^{\infty} \frac{\left(\mp \frac{2}{\Lambda}\right)^{n}}{n !} \chi^{(n)}(0) \frac{\partial^{n}}{\partial z^{n}} \int_{0}^{\infty} \frac{d t}{t} e^{\left[\mp \frac{1}{2} t \Lambda z\right]} \tilde{\rho}\left(t^{2} \Lambda^{2}\right) .
$$

\footnotetext{
${ }^{\mathrm{d}}$ The PU test functions are such that any power of $\tilde{\rho}$ is an equivalent $\mathrm{PU}^{21}$ (Appendix B). Hence, $\tilde{\rho}^{2} \simeq \tilde{\rho}$.
} 
The remaining $t$-integrals with $\tilde{\rho}\left(t^{2} \Lambda^{2}\right)$ and $\Re(z) \lessgtr 0$ defines the Laplace transform of the pseudo-function distribution ${ }^{17} \mathcal{P} f\left(\frac{1}{t}\right)=\frac{d}{d t} \ln (t)$ (cf. Appendix), that is

$$
\mathcal{L}\left\{\mathcal{P} f\left(\frac{1}{t}\right)\right\}=-\ln \left[\gamma_{E}+\left(\frac{z \Lambda}{2}\right)\right]=-\ln \left(\tilde{\Lambda}_{c} z\right) .
$$

It is clear that the contribution with $n=0$ just gives a non-analytic term in $\ln z$ whereas derivatives of higher-order in $z$ generate the Laurent series in $\frac{1}{n} \cdot \frac{1}{z^{(n)}}$, for $\frac{\partial^{n}}{\partial z^{n}} \ln (z)=(-1)^{n-1} \frac{(n-1) !}{z^{(n)}}$. Due to the presence of the nonzero arbitrary scale $\Lambda_{c}$ it is always liable to extract a constant zero-mode contribution $a_{0}$ by writing $\tilde{\Lambda}_{c}=$ $\exp \left(-\frac{a_{0}}{2}\right) \tilde{\Lambda}_{c}^{\prime}$, so that after quantization, the expression for $\phi(z, \bar{z})$ is in agreement with the one given in Refs. 7 and 8 as

$$
\phi(z, \bar{z})=a_{0}-\imath \pi_{0} \ln (z \bar{z})+\frac{\imath}{4 \pi} \sum_{n \neq 0} \frac{1}{n}\left(c_{n} z^{-n}+\bar{c}_{n} \bar{z}^{-n}\right) .
$$

We now proceed to the canonical quantization through the correspondence $\chi\left( \pm p^{+}\right) \curvearrowright\left\{a\left(p^{+}\right)\right.$viz. $\left.a^{\dagger}\left(p^{+}\right)\right\}$and commutator $\left[a(p), a^{\dagger}(q)\right]=(4 \pi)^{2} p \delta(p-q)$ and calculate the two-point vacuum expectation value $(\mathrm{VeV})$ function of the convoluted OPVD fielde with the result

$$
\begin{aligned}
\langle 0|\hat{\varphi}(z) \hat{\varphi}(w)| 0\rangle & =\int_{0}^{\infty}\left[d t \frac{d}{d t} \ln (t)\right] e^{\left[-\frac{1}{2} t \Lambda(z-w)\right]} \tilde{\rho}^{2}\left(t^{2} \Lambda^{2}\right) \\
& =-\frac{1}{2} \ln \left[|z-w|^{2} \tilde{\Lambda}_{c}^{2}\right]
\end{aligned}
$$

with $\tilde{\Lambda}_{c}^{2}=\frac{e^{\gamma_{E}}}{2} \Lambda(\equiv 1)$. In addition

$$
\begin{aligned}
\left\langle 0\left|\partial_{z} \hat{\varphi}(z) \partial_{w} \hat{\varphi}(w)\right| 0\right\rangle & =\partial_{z} \int_{0}^{\infty} d t e^{\left[-\frac{1}{2} t \Lambda(z-w)\right]} \tilde{\rho}^{2}\left(t^{2} \Lambda^{2}\right) \\
& =\partial_{z} \frac{1}{z-w}=-\frac{1}{(z-w)^{2}}
\end{aligned}
$$

To close the comparison, we must check that the $\mathrm{VeV}\langle 0|T(z) T(w)| 0\rangle$ agrees also with the CFT result and thus does characterize the conformal symmetry content of the massless scalar QFT. Knowing the $\operatorname{VeV}\langle 0|\hat{\varphi}(z) \hat{\varphi}(w)| 0\rangle$, the exercise is simple. $\stackrel{8}{ }$ We have directly

$$
\begin{aligned}
\langle 0|T(z) T(w)| 0\rangle & =2\left(\partial_{z} \partial_{w}\langle 0|\hat{\varphi}(z) \hat{\varphi}(w)| 0\rangle\right)^{2} \\
& =\frac{1}{2(z-w)^{4}} \\
& \equiv \frac{1}{12 z^{2} w^{2}} \sum_{n=2}^{\infty} n\left(n^{2}-1\right)\left(\frac{w}{z}\right)^{n} .
\end{aligned}
$$

eIf $|z|>|w|$, the integral is convergent and $\tilde{\rho}^{2}\left(t^{2} \Lambda^{2}\right)$ may be taken to unity over the whole integration domain. On the other hand, the interchange $p \leftrightarrow-p$ in the integral leads to the same result. 
These relations (54)-(56) are precisely the results given in Refs. 7-9 from the Laurent mode expansion.

A consequence of the occurrence of Eq. (53) from our continuum formulation is that the operators $c_{n}$ of the Laurent mode expansion can be equally recovered from contour integration involving the OPVD $\partial \hat{\varphi}(z)$ obtained from Eq. (52)

$$
c_{l}=\frac{1}{2 \pi \imath} \oint d z z^{l} \partial_{z} \hat{\varphi}(z), \quad l \in \mathbb{Z}
$$

Hence, the $c_{l}$ s and the $a(p)$ s have the same vacuum state $|0\rangle$.

The holomorphic tensor $T(z)$ is given by ${ }^{7-\underline{9}}$

$$
T(z)=-\frac{1}{2}: \partial_{z} \hat{\varphi}(z) \partial_{z} \hat{\varphi}(z):=\sum_{n \in \mathbb{Z}} \frac{L_{n}}{z^{n+2}} .
$$

Knowing $T(z)$ from the first equation above, and with

$$
L_{m}^{+}=\frac{1}{2 \pi \imath} \oint d \zeta \frac{T(\zeta)}{\zeta^{m-1}}
$$

it is easy to relate the $\mathrm{VeV}\langle 0|T(z) T(w)| 0\rangle$ with Eq. (56) to the value of the commutator $\left[L_{m}, L_{n}\right]$, showing then its characteristic Virasoro form with central charge unity.

The construction of the massless LF fermion fields proceeds in a completely equivalent manner. The derivation is in a sense simpler, for there is no infrared divergence, but one has to take care of the constraint for the non-dynamical fermion fields component. ${ }^{25}$

\subsection{CFT analysis from discretized light-cone quantization $(D L C Q)$}

Early in the history of LF analysis, DLCQ was advocated ${ }^{16}$ as a method to investigate the peculiarities in relation with this choice of frame. It was soon realized that a major problem was a proper treatment of zero modes and the appearance of infrared divergences in the component $p^{+}$of the momentum $p^{\mu}=\left(p^{+}, p^{-}\right)$. DLCQ fails when taking the limit $p^{+} \rightarrow 0$. This operation has a fundamental relevance in the understanding of the non-perturbative aspects of the field theory under consideration. Here, we shall show what lessons the continuum approach of the preceding section teaches to cure the usual DLCQ caveats.

The discretization of the continuum expansion is usually made through a Fourier series analysis of the classical scalar field, say the classical counterpart of $\tilde{\phi}\left(x^{-}\right)$in Eq. (38), in terms of a set of functions, such as $e^{\left( \pm \imath \frac{n \pi x^{-}}{L}\right)}$, periodic over a characteristic length $L$. The zero mode corresponds to the integral over $x^{-}$in Eq. (38) and it is immediately clear that the expansion coefficient corresponding to this zero mode is ill-defined in the absence of the test function. Hence, as expected, only the modes with $n \geq 1$ enter in the analysis. The non-analytic ln terms have to be interpreted as the signature of the proper limit $n \rightarrow 0$, that is, $p^{+}=\frac{n \pi x^{-}}{L} \rightarrow 0$ in the integral of Eq. (38). These ln terms correspond to a correct treatment of the IR divergence 
mentioned above. They must be taken into account beforehand to have a complete Fourier description of $\tilde{\phi}\left(x^{ \pm}\right)$.

The additional Fourier series can be brought to the forms

$$
\begin{aligned}
& \sum_{n \in \mathbb{Z}} \phi\left(n, x^{+}\right)=\frac{1}{4 \pi} \sum_{n= \pm 1, \pm 2, \ldots} \frac{1}{|n|} \bar{c}_{n} e^{-i \frac{\pi}{L} n x^{+}}, \\
& \sum_{n \in \mathbb{Z}} \phi\left(n, x^{-}\right)=\frac{1}{4 \pi} \sum_{n= \pm 1, \pm 2, \ldots} \frac{1}{|n|} c_{n} e^{-i \frac{\pi}{L} n x^{-}},
\end{aligned}
$$

where

$$
\left[c_{m}, c_{n}\right]=\left[\bar{c}_{m}, \bar{c}_{n}\right]=(4 \pi)^{2} n \delta_{m,-n}, \quad\left[c_{m}, \bar{c}_{n}\right]=0 .
$$

It is important to realize that these expressions are OPVD which cannot be disentangled from their convolution with test functions. This is a second caveat in the usual handling of these Fourier mode expansions. With (59) and (60) completed with the non-analytic ln terms, one has an easy access to correlation functions computed in the continuum theory. But this is where the caveat just mentioned shows up.

Consider then the $\mathrm{VeV}\langle 0|\phi(\mathbf{x}) \phi(\mathbf{y})| 0\rangle$ evaluated in Euclidean coordinates $\{\mathbf{x}, \mathbf{y}\}$. It is the sum of two contributions, the first one coming from the nonanalytic $\ln (z \bar{z})$ terms which is just

$$
-\ln \left[|\mathbf{x}-\mathbf{y}|^{2} \Lambda^{2}\right]
$$

and a second piece coming from Eq. (59) (viz. Eq. (60)) and reducing to $\left(z^{+}=\right.$ $\left.x^{+}-y^{+}\right)$

$$
\lim _{N \rightarrow \infty} \sum_{n=1}^{N} \frac{1}{n} e^{-i \frac{\pi}{L} n\left(z^{+}\right)} .
$$

When $z^{+}=0$, this series diverges like $\ln N$ when $N \rightarrow \infty$. This is, as expected, the signature of a UV-divergence resulting from an ill-defined product of distributions at the same point. The evaluation of the series $(63)$ cannot be done without implementing the test function method in the UV which, in this regime, was shown ${ }^{20,21}$ not to be achieved by the introduction, in the exponential, of a simple converging factor in $-\imath \epsilon$. To summarize Sec. 4.1 of Ref. 21 , the subtraction method can be applied at the level of propagators that consist of subtracting from (63) the same sum with the "mass term" $\frac{1}{L}$ replaced by $\frac{\eta}{L}$, with $\eta>1$ being the intrinsic renormalization group $(\mathrm{RG})$ scale parameter present in the original PU-test function. In effect, we are left with $\left(q=e^{-\frac{2 \pi z^{+}}{L}}\right)$

$$
\begin{aligned}
\sum_{n=1}^{\infty} \frac{q^{n}-q^{n \eta}}{n} & =\ln (1-q)-\ln \left(1-q^{\eta}\right) \\
& =\ln (\eta)+\mathcal{O}\left(\frac{1}{L}\right) .
\end{aligned}
$$


This additional RG-term is not a surprise, for the mass scale is arbitrary, that is $\Lambda \equiv \eta \Lambda$. Hence, it does not change the first contribution to the $\mathrm{VeV}$ in Eq. (62) coming from the non-analytic ln terms. However, it is important to note that, even though IR and UV divergences are both logarithmic, their mathematical treatments are of different nature, for they belong to orthogonal subspaces. It is a key issue in the RG analysis of gauge invariant QFTs. ${ }^{24}$

Turning to the components of the energy-momentum tensor in the discrete representation, they read with $K=-\frac{\pi}{L^{2}}$ as:

$$
\begin{aligned}
& T^{++}\left(x^{-}\right)=K \sum_{m, n} \operatorname{sign}(m) \operatorname{sign}(n): c_{m} c_{n}: e^{-i \frac{\pi}{L}(n+m) x^{-}}, \\
& T^{--}\left(x^{+}\right)=K \sum_{m, n} \operatorname{sign}(m) \operatorname{sign}(n): \bar{c}_{m} \bar{c}_{n}: e^{-i \frac{\pi}{L}(n+m) x^{+}} .
\end{aligned}
$$

These components can be transformed to a "Virasoro form" by simply taking a Fourier transform. Indeed, assume that $T^{++}\left(x^{-}\right)$can be represented as

$$
T^{++}\left(x^{-}\right)=\frac{1}{4 L^{2}} \sum_{l=0, \pm 1, \ldots} L_{l} e^{-i \frac{\pi}{L} l x^{-}} .
$$

The operator coefficients $L_{l}$ are then obtained by inverting this relation:

$$
L_{l}=2 L \int_{-L}^{+L} d x^{-} e^{i \frac{\pi}{L} l x^{-}} T^{++}\left(x^{-}\right) .
$$

In particular,

$$
L_{0}=4 L P^{+}
$$

Inserting $T^{++}\left(x^{-}\right)$in the Fock form (64) into (67) gives

$$
L_{n}=-4 \pi \sum_{k= \pm 1, \ldots} \operatorname{sign}(k) \operatorname{sign}(n-k) c_{k} c_{n-k} .
$$

A lengthy but straightforward calculation ${ }^{25}$ based on the commutators (61) yields the light-front version of the Virasoro algebra, including the $c$-number term, not present at the classical level (the "central extension"),

$$
\left[L_{n}, L_{m}\right]=(n-m) L_{n+m}+\frac{c}{12} n\left(n^{2}-1\right) \delta_{n+m, 0},
$$

where $c$ is the "central charge", equal to 1 . The same procedure applies to the component $T^{--}\left(x^{+}\right)$, leading to the algebra (70) with $L_{n} \rightarrow \bar{L}_{n}$. Obviously, one has $\left[L_{n}, \bar{L}_{m}\right]=0$, since $\left[c_{n}, \bar{c}_{m}\right]=0$.

This confirms the complete conformal content of the free scalar field theory in the Light Front formulation. 


\section{Discussions and Conclusions}

We have shown that the 2D-massless scalar field in the front form of QFT can be recovered in a simple way as the zero mass limit of the corresponding massive field theory and thereby consistently quantized without any loss of physical information. Past tentative attempts are numerous and comparison on an individual basis is tantamount to Hercules' works. In this respect the "Commentaries on the Bibliography and References" of Bogolubov et al.'s Monograph ${ }^{18}$ relative to Chapters 10 and 11 are still relevant. To focus on the essentials of our presentation with respect to most of the past attempts, we may pinpoint the following aspects.

- The nature of the classical solutions to the wave equation in normal spacetime coordinates and LF variables was a long debated problem, for the time propagation in the first set of coordinates seemed to conflict with the implied propagation on the two characteristics of the second set. This observation hindered canonical quantization in the LF variables and even lead to erroneous attempts to include separate wave propagation on the two characteristics. We have shown clearly that there is no such conflict. This finding is a prerequisite for the validation of canonical quantization in LF variables which appear naturally in D'Alembert's classical solution.

- The second major difficulty encountered and recognized by all authors is the occurrence of an infrared divergence in the conjugate LF variable $p^{+}$, which hinders the construction of a massless scalar field theory with fields as regular operator-valued distributions in a Hilbert space. The treatment of this divergence varies from an $a d$-hoc suppression with an infrared cutoff $\grave{a}$ la Klaiber ${ }^{26,27}$ to mathematical considerations based on the theory of distributions, where the standard approach in the literature removes the divergence with the Cauchy principal value of $\frac{1}{p^{+}}$. Our approach is based on the mathematics of the extension of singular distributions in the infrared explained in our earlier publications. Its basis is the existence of PU test function with genuine limiting properties and leads for the infrared to the notion of pseudo-functions specific to Schwartz's formulation ${ }^{17}$ of distribution theory. A good testing ground for the power of this method was the successful resummation ${ }^{28}$ of the infrared divergent infinite perturbative mass expansion of the known massive field propagator in dimension $d=2$ (which is hopeless without the use of $\mathcal{C}^{\infty} \mathrm{PU}$ test functions).

- No recourse is necessary to axiomatic assumptions and presupposed commutators of fields. Our only requirement is that classical fields have to live on differential manifolds with the associated calculus on differential forms. This property occurs as a consequence of the convolution operation of classical fields distributions with PU test functions.

- The construction of the Hilbert space necessitates the existence of the norm of the one particle state $\|\left. a_{\tilde{\rho}}^{+}\right|_{\Omega}|0\rangle \|_{L^{2}(\Omega)}^{2}=\int_{\Omega} d X \frac{\tilde{\rho}(X)}{X}$ with $\tilde{\rho}(X) \in \Omega \subset \mathbb{R}^{+}$. This integral is bounded when defined in terms of a PU (cf. Appendix). The full Hilbert space of states (Fock space) is generated via the standard mathematical 
procedure of linear superposition and Cauchy's completion.

- Even though the fundamental CFT framework in two dimensions was known ${ }^{6}$ as early as 1984, major contributions on canonical quantization of the scalar massless field around this period, and surprisingly beyond, paid at most a nominal attention to the consequences of conformal symmetry. For instance, in Bogolubov et al.'s Monograph, $\frac{18}{2}$ the two-point function of Eq. (11.12) exhibits the same structure as our result of Eq. (54), but the link with the CFT result is not acknowledged as such. On the other hand, the noticeable contribution of Morchio et al. ${ }^{29}$ is more talkative with respect to conformal transformations. However, it does not come to our comparison of canonical LF quantization and CFT formulation.

The main result of this work is genuine in that, our canonical formalism correctly incorporates the features of the underlying conformal symmetry. We have established a direct link between the two formulations simply by going over to the Euclidean time in the correlation functions calculated within the LF framework. The Virasoro algebra was also derived in a straightforward manner by Fouriertransforming the components of the massless LF energy-momentum tensor. Additional conformal properties of the LFT including treatment of the massless fermion field will be published separately. ${ }^{25}$

Let us emphasize finally that the consistency of the procedure with the usual canonical quantization scheme in terms of initial field values in the Minkowski time can only be recovered when fields are considered as operator-valued distributions with PU test functions $\tilde{\rho}$ such that for the light-cone momentum $p^{+}$, $\lim _{p^{+} \rightarrow 0^{+}} \frac{\tilde{\rho}\left[\left(p^{+}\right)^{2}\right]}{p^{+}}=0$. Thereby, the Light Front Formulation and Conformal Field Theory analysis of 2D-massless models are in complete agreement.

\section{Appendix A. Properties of the PU Test Function in the Limit $\alpha \rightarrow 1^{-}$}

In the small $X$ region (IR), it is easy to see that the condition

$$
\lim _{\alpha \rightarrow 1^{-}} g_{\alpha}(X)=1+\mathcal{O}((\alpha-1) X),
$$

leads to the lower end of the integration domain when solving the two equations $X=2 h(X)$ and $X=h(X)$ for small $X$. Indeed, the solutions are, respectively, $X 01=\frac{2(1-\alpha)}{2 \eta^{2}-1}$ and $X 02=\frac{(1-\alpha)}{\eta^{2}-1}$, which both tend to $0^{+}$when $\eta^{2}>1$ and $\alpha \rightarrow 1^{-}$. X01 being the lower boundary of the PU-support, the lower limit of the $X$-integration tends to zero, as it should. Moreover, and whatever the functional form of $u(X)$, the resulting PU behaves like a smeared $\theta$-function ${ }_{-}^{\mathrm{f}}$ in the IR, with vanishing derivatives in $X 01$ and $X 02$ to all order. In the large- $X$ limit (UV),

\footnotetext{
${ }^{\mathrm{f}}$ Figure 6 of Ref. 22 shows a PU set-up for a particular functional choice of the elementary function
} $u(X)$. 
any form of $g_{\alpha}(X)$ satisfying the condition (A.1) will do and we keep the choice made earlier in Refs. 22 and 24, that is $g_{\alpha}(X)=X^{(\alpha-1)}$. Other choices only modify the rate at which the upper boundary of the PU-support goes to infinity when $\alpha$ approaches $1^{-}$, the extended distribution being the same, as it should. Solving again the two equations $X=h(X)$ and $X=2 h(X)$ in the large- $X$ limit - where $(\alpha-1)$ can be neglected in $(15)$, the solutions are, respectively, $X 11=\left(\eta^{2}\right)^{\left(\frac{1}{1-\alpha}\right)}$ and $X 12=\left(2 \eta^{2}\right)^{\left(\frac{1}{1-\alpha}\right)}$. For $\eta^{2}>1$, both values tend to infinity when $\alpha \rightarrow 1^{-}$and the PU-support stretches to cover the whole domain of integration in $X$. All those features are generic of the PU set-up since they are independent of the functional form of $u(X)$ and of the value of the scale parameter $\eta>1$ governing the shape ${ }^{\mathrm{f}}$ of $\beta_{1}$ and $\beta_{2}$ and the size of their respective support.

\section{Appendix B. Definition and Properties of the Pseudo-Function Distributions}

Recall on $\mathcal{P} f\left(\frac{1}{x}\right)$ : One defines

$$
\frac{1}{x_{+}}=\frac{1}{x} \quad \text { for } x>0 ; \quad 0 \text { for } x<0
$$

and

$$
\left\langle\mathcal{P} f\left(\frac{1}{x_{+}}\right), \rho\right\rangle=\lim _{\epsilon \rightarrow 0}\left[\int_{\epsilon}^{\infty} \frac{\rho(x)}{x} d x+\rho(0) \ln (\epsilon)\right],
$$

in the same way

$$
\left\langle\mathcal{P} f\left(\frac{1}{x_{-}}\right), \rho\right\rangle=\lim _{\epsilon \rightarrow 0}\left[\int_{-\infty}^{-\epsilon} \frac{\rho(x)}{x} d x-\rho(0) \ln (\epsilon)\right] .
$$

Hence,

$$
\text { Cauchy PV: } \mathcal{P} \mathcal{V}\left(\frac{1}{x}\right)=\mathcal{P} f\left(\frac{1}{x_{+}}\right)+\mathcal{P} f\left(\frac{1}{x_{-}}\right) .
$$

In the context of distributions, one defines

$$
\ln \left(x_{+}\right)=\ln (x) \text { for } x>0 ; \quad 0 \text { for } x<0
$$

and

$$
\left\langle\ln \left(x_{+}\right), \rho\right\rangle=\int_{-\infty}^{\infty} \ln \left(x_{+}\right) \rho(x) d x=\int_{0}^{\infty} \ln (x) \rho(x) d x,
$$

which gives (definition of the derivative of a distribution)

$$
\begin{aligned}
\left\langle\frac{d}{d x} \ln \left(x_{+}\right), \rho\right\rangle & =\lim _{\epsilon \rightarrow 0} \int_{\epsilon}^{\infty} \ln \left(x_{+}\right)\left(-\rho^{\prime}(x)\right) d x, \\
& =\lim _{\epsilon \rightarrow 0}\left[\rho(\epsilon) \ln (\epsilon)+\int_{\epsilon}^{\infty} \frac{\rho(x)}{x} d x\right],
\end{aligned}
$$


that is

$$
\left\langle\frac{d}{d x} \ln \left(x_{+}\right), \rho\right\rangle=\lim _{\epsilon \rightarrow 0}\left[\int_{\epsilon}^{\infty} \frac{\rho(x)}{x} d x+\rho(0) \ln (\epsilon)+\epsilon \rho^{\prime}(\eta) \ln (\epsilon)\right] .
$$

As $\epsilon \ln (\epsilon) \rightarrow 0$ with $\epsilon$ and since $\rho^{\prime}(\eta)$ stays bounded, one concludes

$$
\frac{d}{d x} \ln \left(x_{+}\right)=\mathcal{P} f\left(\frac{1}{x_{+}}\right) ; \quad \frac{d}{d x} \ln \left(x_{-}\right)=\mathcal{P} f\left(\frac{1}{x_{-}}\right) .
$$

It has been pointed out ${ }^{18,29}$ that structural properties of gauge field theories have interesting imbrications with massless fields in two dimensions. The definition of the pseudo-function is actually subject to a certain scale arbitrariness ${ }^{17}$ (cf. Chap. II, p. 41) which is tantamount to a freedom in the gauge choice.

Indeed $\forall a>0 \in \mathbb{R} \int_{0}^{a} d x \mathcal{P} f\left(\frac{1}{x}\right) \doteq \lim _{\epsilon \rightarrow 0} \int_{\epsilon}^{a} \frac{d x}{x}+\log \epsilon=\log (a) \equiv \lim _{\epsilon^{\prime} \rightarrow 0} \int_{\epsilon^{\prime}}^{1} \frac{d x}{x}+$ $\log \epsilon^{\prime}$ with $\epsilon^{\prime}=a \epsilon$. For Abelian and Yang-Mills gauge theories, the scale parameter $a$ is indeed directly connected to the gauge choice. ${ }^{24}$

\section{Appendix C. On the Norm of the One Particle State in Terms of the Pseudo-Function $\mathcal{P} f\left(\frac{1}{x_{+}}\right)$}

This norm is given by the integral

$$
\begin{aligned}
I_{N} & =\int_{0}^{\infty} d X \frac{\tilde{\rho}(X)}{X} \\
& =\left[\int_{0}^{\Lambda} \frac{d X}{X} \tilde{\rho}(X)+\int_{0}^{\frac{1}{\Lambda}} \frac{d X}{X} \tilde{\rho}\left(\frac{1}{X}\right)\right],
\end{aligned}
$$

here, both $\tilde{\rho}(X)$ and $\tilde{\rho}\left(\frac{1}{X}\right)$ have the necessary properties ${ }^{21}$ leading to the pseudofunction distribution extension $\mathcal{P} f\left(\frac{1}{X}\right)$ of $\frac{1}{X}$ at the origin, however with different arbitrary scalings, one, $a$, coming from the initial IR region, and another, $\eta^{2}>1$, coming from the original UV region. Its extension is governed by the scale parameter $\eta$ inherent in the construction of the PU, with value $\left(\eta^{2}\right)^{\left(\frac{1}{1-\alpha}\right)} \equiv \eta^{2}\left(\eta^{2}\right)^{\left(\frac{\alpha}{1-\alpha}\right)}$. It tends to infinity when $\alpha \rightarrow 1^{-}$. By the change of integration variable $X \curvearrowright \frac{1}{X}$, it is transformed to $\frac{\epsilon}{\eta^{2}}$ with $\epsilon=\lim _{\alpha \rightarrow 1^{-}}(1-\alpha)$. There is no gauge consideration at this stage and $a=1$. Thus, $I_{N}$ becomes

$$
\begin{aligned}
I_{N} & =\lim _{\epsilon \rightarrow 0}\left[\int_{\epsilon}^{\Lambda} \frac{d X}{X}+\int_{\frac{\epsilon}{\eta^{2}}}^{\frac{1}{\Lambda}} \frac{d X}{X}+2 \log \epsilon\right], \\
& =\log \eta^{2} .
\end{aligned}
$$

\section{Acknowledgments}

We acknowledge the financial support from CNRS/IN2P3-Department during the course of this work. E.W. is grateful to Denis Puy for his kind hospitality at the LUPM. 


\section{References}

1. P. A. M. Dirac, Rev. Mod. Phys. 21, 392 (1949).

2. P. A. M. Dirac, Mathematical Foundation of Quantum Theory (Academic Press, 1978).

3. (Eds.) P. Grangé, H. C. Pauli, A. Neveu, S. Pinsky and E. Werner, New NonPerturbative Methods and Quantization on the Light Cone, Les Houches Series, Vol. 8 (Springer, 1998).

4. P. A. M. Dirac, Can. J. Phys. 1, 1 (1950); iibid., Lectures on Quantum Mechanics (Benjamin, 1964); J. L. Anderson and P. G. Bergmann, Phys. Rev. 83, 1018 (1951); P. G. Bergmann, Helv. Phys. Acta Suppl. IV, 79 (1956); K. Sundermeyer, Constrained Dynamics, Lecture Notes in Physics, Vol. 169 (Springer, 1982).

5. L. Faddeev and R. Jackiw, Phys. Rev. Lett. 60, 1692 (1988); R. Jackiw, (Constrained) Quantization Without Tears, MIT preprint CTP 2215 (1993), arXiv:hep-th/9306075.

6. A. A. Belavin, A. M. Poliakov and A. B. Zamolodchikov, Nucl. Phys. B 241, 333 (1984).

7. P. Di Francesco, P. Mathieu and D. Sénéchal, Conformal Field Theory, Grad. Texts in Contemporary Physics (Springer-Verlag, 1997).

8. C. Itzykson and J. M. Drouffe, Théorie Statistique des Champs, Savoirs Actuels, Inter Editions, Vol. 2 (Editions du CNRS, Paris, 1989).

9. Y. Grandati, Ann. Phys. (Paris) 17, 159 (1992).

10. S. Weinberg, Phys. Rev. 150, 1313 (1966).

11. H. Leutwyler, Springer Tracts Mod. Phys. 50, 29 (1969).

12. R. A. Neville and F. Rohrlich, Nuovo Cimento A 1, 625 (1971).

13. J. B. Kogut and D. E. Soper, Phys. Rev. D 1, 2901 (1971).

14. S.-J. Chang, R. G. Root and T.-M. Yan, Phys. Rev. D 7, 1133 (1973).

15. H. Leutwyler, J. R. Klauder and L. Streit, Nuovo Cimento A 66, 536 (1970).

16. H. C. Pauli and S. J. Brodsky, Phys. Rev. D 32, 1993 (1985); 32, 2001 (1985).

17. L. Schwartz, Théorie des Distributions (Hermann, 1966).

18. N. N. Bogolubov, A. A. Luganov, A. I. Oksak and I. T. Todorov, General Principles of Quantum Field Theory (Kluwer Publishers, 1990).

19. K. Osterwalder and R. Schrader, Commun. Math. Phys. 31, 83 (1973).

20. P. Grangé, P. Ullrich and E. Werner, Phys. Rev. D 57, 4981 (1998).

21. P. Grangé and E. Werner, J. Phys. A: Math. Theor. 44, 385402 (2011); Nucl. Phys. (Proc. Suppl.) B 161, 75 (2006).

22. P. Grangé, J.-F. Mathiot, B. Mutet and E. Werner, Phys. Rev. D 80, 105012 (2009).

23. T. Heinzl and E. Werner, Z. Phys. C 62, 521 (1994).

24. P. Grangé and E. Werner, J. Phys. A: Math. Theor. 45, 315401 (2012).

25. L. Martinovic, private communication.

26. B. Klaiber, The thirring model, in eds. A. O. Barut and W. E. Brittin, Lectures in Theoretical Physics, Vol. 10A (Gordon and Breach, 1968), pp. 141-176.

27. N. Nakanishi, Prog. Theor. Phys. 57, 269 (1977).

28. P. Grangé and E. Werner, Nucl. Phys. (Proc. Suppl.) B 161, 75 (2006).

29. G. Morchio, D. Pierotti and F. Strocchi, J. Math. Phys. 31, 1467 (1990). 